\title{
Detailed distributions of the CO
}

\section{$J=(2-1) / J=(1-0)$ intensity ratios toward a large area of the central molecular zone}

\author{
Kazufumi Torii ${ }^{1}$, Rei Enokiya ${ }^{1}$, Yasuo Fukui ${ }^{1}$, Hiroaki Yamamoto ${ }^{1}$, \\ Akiko Kawamura ${ }^{2}$, Norikazu Mizuno ${ }^{2}$, Toshikazu Onishi ${ }^{3}$ and \\ Hideo Ogawa ${ }^{3}$ \\ ${ }^{1}$ Department of Physics, Nagoya University, Furo-cho, Chikusa-ku, Nagoya, Aichi 464-8601, \\ Japan; email: torii@a.phys.nagoya-u.ac.jp \\ ${ }^{2}$ National Astronomical Observatory of Japan, Mitaka, Tokyo 181-8588, Japan \\ ${ }^{3}$ Department of Astrophysics, Graduate School of Science, Osaka Prefecture University, 1-1 \\ Gakuen-cho, Naka-ku, Sakai, Osaka 599-8531, Japan
}

\begin{abstract}
We present the first results of the new CO $J=(2-1)$ observations toward the central molecular zone (CMZ) using the NANTEN2 telescope at an angular resolution of 100". Large area coverage of $4^{\circ} \times 2^{\circ}$ in $l$ and $b$ and a high angular resolution of $100^{\prime \prime}$ enable us to investigate detailed structures of the molecular gas in the CMZ including peculiar molecular filaments perpendicularly to the Galactic plane to $b>\left|0.5^{\circ}\right|$. The major components of the CMZ, e.g., Sgr A, Sgr B and Sgr C cloud complexes, show high CO $J=(2-1) / J=(1-0)$ ratios around 0.9 , indicating highly excited conditions of the molecular gas, while the local foreground components show less than 0.4. The molecular filaments show the typical ratios of $0.6-0.7$ indicate that they are indeed located in the Galactic center.
\end{abstract}

Keywords. ISM: clouds - Radio lines: ISM — Galaxy: Galactic center

\section{Introduction}

In the Galactic center (GC), CO (Carbon Monoxide) rotational transitions have been used to investigate a huge molecular cloud complex, the central molecular zone (CMZ), distributed at the inner $300 \mathrm{pc}$ of the Galaxy. Recent infrared observations covering a large area of the CMZ reveal the existence of the striking structures with sub-pc to pc scales at the high latitude area of the GC $\left(b>\left|0.5^{\circ}\right|\right)$, such as the Double Helix nebula (DHN; Morris et al. 2006). On the other hand, the past observations of the molecular transitions with spatial resolutions comparable to these infrared observations are limited to the low latitude region of the CMZ $\left(b<\left|0.3^{\circ}\right|\right)$, making it difficult to make direct comparisons with these infrared structures. We have carried out new ${ }^{12} \mathrm{CO} J=(2-1)$ observations toward $4^{\circ} \times 2^{\circ}$ of the CMZ using the NANTEN2 4-m telescope situated at Atacama, Chile. It provided a spatial resolution of $100^{\prime \prime}$, corresponding to $\sim 4$ pc at the GC. The observations fully cover the infrared structures at the high latitude region.

\section{Results}

Figure 1 shows the velocity channel maps of the ${ }^{12} \mathrm{CO} J=(2-1)$ integrated intensities in contours and the ${ }^{12} \mathrm{CO} J=(2-1) / J=(1-0)$ ratios in color. The ${ }^{12} \mathrm{CO} J=(1-0)$ data used here is a part of the NANTEN Galactic survey (Takeuchi et al. 2010). Our observations resolve the major cloud complexes of the CMZ, such as Sgr A, Sgr B2 and 


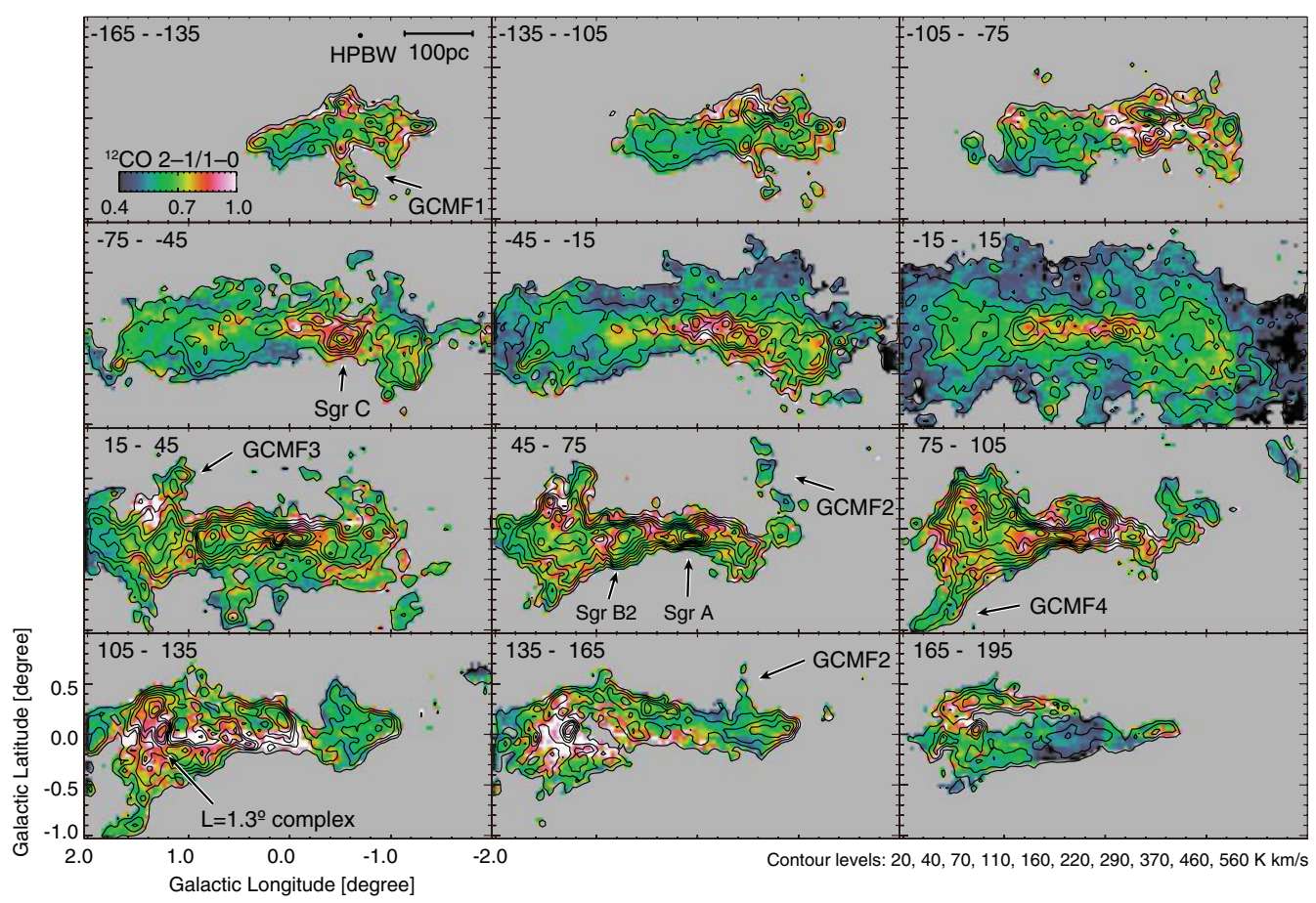

Figure 1. Velocity channel maps of the ${ }^{12} \mathrm{CO} J=(2-1)$ integrated intensities (contours) and ${ }^{12} \mathrm{CO} J=(2-1) / J=(1-0)$ ratios (color). [A COLOR Version is AVAilable onLine.]

Sgr C, into individual clouds. They show relatively high ratios of about 0.9 and up to over 1.0, while those of the foreground gas not related to the GC is only 0.4. The high ratio over 1.0 indicates that the ${ }^{12} \mathrm{CO}$ lines are optically thin. The molecular clump CO $1.27+0.01$ in the " $l=1.3^{\circ}$ complex" shows the highest ratios among all clouds, where the existence of the strong shock is indicated (Tanaka et al. 2007).

The filamentary structures (hereafter we refer as GC molecular filaments (GCMF)) mainly have their foot points around the eastern and western ends of the CMZ. Only the molecular ridge associated with the infrared emission of the DHN detected by Enokiya et al. (2014) has its foot point around Sgr A* (it is distributed around $v=0 \mathrm{~km} \mathrm{~s}^{-1}$ ). GCMF 1 has infrared counterparts that are similar to the molecular ridge of the DHN, and GCMF 2, which consists of two filaments, is spatially correlated to the extended infrared emission AFGL 5376 (Uchida et al. 1990). GCMF 3 was discussed by Sofue (2007) that it is a molecular tornado driven by a magnetic squeezing mechanism. GCMF 4 looks like a southern counterpart of GCMF 3. These two have no clear counterparts in the infrared. The typical $J=(2-1) / J=(1-0)$ ratios of the GCMFs are as follows: GCMF $1=0.8$, GCMF $2=0.6$, GCMF $3=0.8$ and GCMF $4=0.7$. All of them have higher ratios than that of the foreground gas of 0.4 , supporting that these four are actually distributed at the GC. Further studies to make comparisons with infrared data sets will be published separately.

\section{References}

Enokiya, R., et al. 2014, ApJ 780, 72

Sofue, Y. 2007, PASJ 59, 189 
Morris, M., Uchida, K., \& Do, T. 2006, Nature 440, 308

Takeuchi, T., et al. 2010, PASJ 62, 557

Tanaka, K., et al. 2003, PASJ 59, 323

Uchida, K., Morris M. \& Serabyn, E. 1990, ApJ 351, 443 